\title{
Author Index Vol. 29, 1995
}

Abstracts in No. 4 have their own Author Index

Aitchison, T.C. 455 Alonzo,G. 285 Amerongen, J.P. van 371 Amerongen, W.E. van 46 AngmarMånsson, B. 2, 20 Arends,J. 14,118,223,231,237

Bashir,E. 467

Beeley,J.A. 111

Beighton, D. 154

Bennett, C.B. 461

Birkhed, D. 54, 172, 337, 402, 435

Bjørndal,L. 243

Blinkhorn, A.S. 163

Bosch, J.J. ten 2, 8

Bowen,W.H. 498,507

Brown, J. 477

Buijs,J.F. 442

Buijs,MJ. 107

Burgersdijk, R.C.W. 272

Burns, T. 192

Carruthers, L.M.C. 424 Cate, J.M. ten 107,371,442 Chen,X.Q. 122 Chestnutt, I.G. 266,455

Christoffersen, J. 223 Christoffersen, M.R. 223 Colby, S.M. 407 Creanor,S.L. 424,477

Creedon,P. 431 Cui,F,Z. 122 Cutress,T.W. 130,258

Dahllöf,G. 343,449 Davies,R.M. 163 Davies, T.G.H. 163 Davis, S. 461 De Crousaz, P. 26

Deery,C. 377 Den Besten, P.K. 251 DeRouen,T.A. 461 Duggal,M.S. 75 Dung, S.-Z. 483

Ekstrand, K.R. 243 Ellwood,R.P. 137 Emilson,C.G. 54,396

Falster,A.U. 285 Featherstone, J.D.B. 143 Filler, S.J. 355 Firestone, A.R. 81,355

Forss,H. 50 Foye,R.H. 424,477 Fyffe,H.E. 377

Geddes, D.A.M. 477 Giambro,NJ. 251 Giertsen,E. 181 Gilmour,W.H. 477 González-Cabezas, C. 198 Graaff, J. de 46 Gregory, R.L. 198,483 Grenby,T.H. 418 Grindeijord, M. 343,449

Guggenheim, B. 382 Guha-Chowdhury, N. 130 Gustavsson, A. 467 Gwinner, L.M. 143

Hall,A.F. 477

Hallgren,A. 188

Hamilton, F.A. 163

Harrington, DJ. 407

Hausen, H. 327

Hawley,G.M. 163

Heaven, T.J. 355

Heilman,J.R. 204

Hintze,H. 359

Hoeven, J.S. van der 159,272

Hof, M.A. van’t 94 
Holbrook, W.P. 42

Holland, T.J. 431

Holloway, P.J. 163

Hotz,P. 81

Hujoel,PJP. 461

Huysmans, M.C.D.NJ.M. 88,100

Ie,Y.L. 94 Iijima,Y. 231 Imfeld,T. 172 Inaba,D. 231 Ireland, A.J. 470 Isokangas, PJ. 461

Isotupa,K.P. 461 Ito,T. 218 Iwami,Y. 130 Iwase, T. 513

Jacobson, A.P.M. 266 Jaeggi, T. 349 Jaeggi-Schärer, S. 349 Jitpukdeebodintra, S. 413 Johnston, D.W. 331 Jones, P.R. 266

Jongebloed, W.G. 118 Jordan, T.H. 204 Josselin de Jong, E. de 2

Kafrawy, A.H. 198 Kambara,M. 210 Kanayama, T. 218 Kato,K. 218 Kippuw, N. 442

Kleber,CJ. 237,490 Köhler,B. 402 Kondo,K. 218 Koontongkaew, S. 413 Kopec, L.K. 507

Kuzmina, I. 243

Lagerlöf,F. 467 Lamont, R.J. 461 Larsen, M.J. 258, 359 Lazarchik, D.A. 355 Legier-Vargas, K. 143 Leonardsen, E.S. 223 Leppänen, T. 327 Leskelä, I. 62 Lewis, D.W. 331 Li,H.D. 122 Li,Y. 198,483 Lincir, I. 168 Lingström, P. 172 Loveren, C. van 442 Lussi,A. 81,349,355 Lynch, E. 154

MacFarlane, T.W. 455 McGuinness, N. 470 Magnúsdóttir, M.O. 42 Mäkinen,K.K. 461

Marthaler, T.M. 26, 35 Menghini, G. 26 Mistry, M. 418 Modéer,T. 343,449 Möttönen,M. 280

Mundorff-Shrestha, S.A. 143

Nakagaki,H. 218 Nakamoto,T. 285 Narita,N. 218 Näse,L. 50 Nilsson,B. 343 Noblitt,T.W. 198

Norde,W. 210 Nugent, Z. 377 Nuttall,N.M. 377

Olsson,S. 402 O’Mullane, D.M. 137,431 Ostela,I. 62 Osterwalder, V. 382 O’Sullivan, D.M. 148

Pape, H.R., Jr. 461 Pearce, E.I.F. 130,258 Pearson, G.J. 192 Penning, Ch. 371 Petersson, L.G. 188 Pienihäkkinen, K. 62 Pitts, N.B. 377 Pollard, M.A. 68,75 Prostak,K. 251 Putt, M.S. 237,490

Raitio,M. 280 Rangmar,B. 435 Rijkom, H.M. van 364 Robinson, C. 218 Roeters, F.J.M. 272

Rondel, P. 88,100 Rosin-Grget, K. 168 Ruben, J. 14,118,231,337 Rudolphy, M.P. 371 Russell,

R.R.B. 407

Saunders, W.P. 424 Schaeken, M.J.M. 94,159,272 Schäfer,F. 266 Scheie, A.Aa. 181 Schneider, P.E. 285 Schoenberg, V. 81 Schüpbach,P. 382 Seppä,L. 50,327 Sherriff,M. 470 Sigurjóns, H. 42 Simmons, W.B. 285 Sjögren,K. 337,435 Söderling, E. 62 Soet, J.J. de 46 Steiner, M. 26 Stephen, K.W. 266,455 Stevenson, A.G. 111 Stich,H. 81 Stokroos,I. 118 Stookey,G.K. 198,483 Strang,R. 424,477 Strijp, A.J.P. van 107 Sundström, F. 2

Tagomori, S. 513 Tahmassebi, J.F. 75 Takagi,O. 231

Tenovuo, J. 1,62 Thibodeau, E.A. 148 Thylstrup,A. 243 Tranaeus, S. 2 Twetman,S. 188

Uhari, M. 280

Vaarkamp, J. 8 Vacca-Smith, AM. 498 Verdonschot, E.H. 8, 88, 94,100, 364

Wang,Q. 122 Warpeha, R.A. 35 Weerheijm, K.L. 46 Wefel,J.S. 204 Wen,H.B. 122

Wennerholm, K. 54, 396 Wenzel,A. 359 Westerling, H. 2 Whelton,H. 431 Whitford, G.M. 20

Wilson, M. 192 Worthington, H.V. 163

Yip,H.K. 111

522

Author Index Vol. 29, 1995 